\title{
Which AQM fits IoT better?
}

\author{
Carlo Augusto Grazia, Natale Patriciello, Martin Klapez, Maurizio Casoni \\ Department of Engineering Enzo Ferrari \\ University of Modena and Reggio Emilia \\ via Pietro Vivarelli 10, 41125 Modena, Italy. \\ \{carloaugusto.grazia, natale.patriciello, martin.klapez, maurizio.casoni\}@unimore.it
}

\begin{abstract}
Most of the Internet traffic is carried by Transmission Control Protocol (TCP) nowadays, even in the case of real-time services and IoT environments. A key point of each TCP variant that defines the specific variant trademark is the congestion control algorithm. This congestion control, alone, is not able to mitigate the congestion problem completely; the most efficient solution, to be coupled with it, is the Active Queue Manager (AQM). In this paper, we analyze the response to congestion provided by the novel AQMs when coupled with popular TCP variants and, has happened in IoT networks, with a mix of TCP variants simultaneously active on the network. We provide extensive simulations within ns-3 to highlight what is the better AQM solution for IoT networks by considering the TCP algorithm and other environmental conditions like the number of active nodes, the network RTT and the presence of multiple RTT flows. We collected network metrics like throughput, goodput, latency, RTT variation, flows' fairness and a simple drop analysis.

Index Terms-AQM, Congestion Control, Cross-Comparison, IoT, QoS, TCP.
\end{abstract}

\section{INTRODUCTION}

Active Queue Management (AQM) was formally introduced in IP networks more than 20 years ago as a viable complementary approach for congestion control, and there has been a steady stream of research output that has, again, come into the spotlight of network operators, maintainers, vendors and OS developers. As a proof of that, CoDel and PIE are two AQM algorithms that have recently been presented and discussed in the IRTF and the IETF as solutions for keeping latency low. These novelties reflect the growing concern and sensitivity about the end-to-end latency perceived by today's Internet users, an outcome of the so-called bufferbloat problem.

As the best of our knowledge, different AQM solutions have been so far only compared, designed and evaluated in the presence of standard TCP New Reno variant. This is a limitation if we consider that TCP is used by most of the current Internet applications, the default TCP on Linux (since version 2.6.19) is Cubic and many other TCP variants has been developed [1]. Moreover, the key characteristic of each TCP variant, which is the trademark that distinguishes one version to another, is the congestion control algorithm. TCP congestion control alone is not suitable for modern heterogeneous networks [2] and is a shared well-known fact in the scientific community that the best cooperation of TCP and AQM is the best treatment of the congestion [3]. Recently, a Google's group developed Bottleneck Bandwidth and Roundtrip (BBR), a novel TCP variant, still under testing currently, designed to identify the bottleneck features and to reduce congestion and latency [4]. It is not near enough to consider congestion nor bufferbloat a case closed.

A challenging scenario is represented by IoT, in which the TCP choice for the end-nodes in not standard and the amount of different TCP traffic leads to a critical congestion problem [5]. This issue gathered the attention of the IoT community that is steering the research against congestion even through IoT subgroup like IoT applications for data centers [6], memory constrained IoT [7] and QoS architecture, in general, for IoT networks [8]. Choosing the right AQM in IoT environment, also considering the TCP equipped by the end-nodes, is fundamental to deal effectively with congestion and performance boosting.

In this paper, we want to show empirically, through extensive simulation analysis, which AQM algorithm suits better IoT networks by considering the TCP used, single or multiple TCP variants simultaneously, and other critical environmental challenges such as long delay, different RTT flows and the number of active flows. We consider promising AQM algorithms, including also new solutions, like ARED [9], CoDel [10], DropTail, GREEN [11], PIE [12] and PINK [13]. We have coupled these AQMs together with many TCP congestion control algorithms, in particular, in this paper, we report only Cubic [14], HighSpeed [15], Hybla [16], Illinois [17], New Reno [18], Vegas [19], Westwood [20] and Yeah [21]. We have chosen these TCPs due to their variety of their mechanisms as well as their availability on ns-3.

The goals are multiple: (i) to check if $\mathrm{AQM}$ algorithms maintain their performance by moving from TCP New Reno to other TCP variants and (ii) to highlight, if it exists, one (or more) AQM(s) able to deal better with congestion in an IoT scenario. We observed the end nodes goodput and throughput, the average queue size and the RTT variation at the bottleneck link, the flows' fairness and the drops occurred.

The rest of the paper is organized in this way: Section II summarizes the related works. Section III introduces briefly the AQM algorithms adopted in this paper, while Section IV describes the environment used for the tests. Section V shows the simulation results of the cross-comparison, and finally, in Section VI the conclusions of our work are drawn.

\section{RELATED WORK}

The AQM world have grown rapidly in the last decades, several version of this algorithms have been proposed in the literature and a detailed survey has been recently reported 
in [22]. As a proof of the continuously evolving topic, an analysis about CoDel and PIE is missing, which are both promising proposals included in our paper.

The problem that we highlighted is the lack of the literature of works studying together AQM and TCP protocols, in particular in the IoT world where there is not a "de facto" standard about the TCP variant to use and it is typical to face great amount connections deploying different TCP congestion controls. Nevertheless, some attempts exist. As an example, an interesting related work is represented by [23], [24] in which a single proposed TCP congestion control, named Compound TCP (C-TCP), has been tested as a function of the AQM algorithm used on the gateway node. Unfortunately, this analysis is limited to the old REM and the simple DropTail AQMs, also considering, in this case, the buffer size as a parameter. Continuing in this direction, another interesting work has been proposed by Khademi et al. in [25]. The authors proposed a comparison between the most promising and novel AQM solutions like CoDel and PIE against Adaptive RED (ARED), a decade-old variant of RED. The paper shows that, in a general access network, the performance of ARED are similar (and in particular cases even better) of the competitors. For what concern the TCP used, this related work investigated only the standard TCP New Reno as the TCP deployed in the end nodes.

By getting closer to the scope of this paper, as the best of our knowledge, only one work has tried to compare AQM algorithms considering different TCP version used in the network, this work is reported in [26]. The environment is similar to the one depicted in [25] but, unfortunately, only two major TCP variants have been considered, i.e. New Reno and Cubic while the paper lacks mainly in the choice of the AQMs tested. Unfortunately, both CoDel and PIE were not available when the paper was written in 2010. Additionally, the figures of merit evaluated in the manuscript have been limited to the throughput achieved by the bottleneck link, together with the average queue occupancy.

Reaching the scope of this paper, only a couple of works highlighted the importance of congestion control for IoT. In [6] the authors proposed an SDN-based TCP named SDTCP, suitable for datacenters configured in an SDN-like way. This work manifests the necessity to take care of congestion control in IoT world due to the amount of the simultaneously active remote services but, unfortunately, the authors considered only datacenters as the testing environment and do not compare the proposed TCP with standard Linux variants. Moreover, the queuing discipline used for their test has been the sole DropTail, which is not enough for an accurate congestion control treatment. An even more focused work is [27] in which the authors described in detail their congestion control mechanism designed for IoT, in particular, they implement a packet discarding technique using WSN architecture knowledge. Unfortunately, even in this case, no state-of-the-art comparison support the results, no cross comparison with $\mathrm{AQM}$ and TCP is provided, and the old ns2 is used for testing platform leading to a lack of transparency.

\section{AQM ALGORITHMS}

In this Section, we briefly describe the algorithms used for our cross-checking comparison. The idea is to provide their core characteristics in a nutshell. We do not include deep descriptions about TCP variants used in our tests, we remind interested readers about TCP mechanisms to a more detailed work aimed to survey this protocol [1].

ARED. Adaptive RED (ARED) [9] is a half decade old variant of RED, that overcome his historical challenge with the deployment, i.e. the tuning of its parameters. This tuning problem has been a discouraging factor for vendors and network operators to deploy RED on congested routers. Despite RED, a characteristic of ARED is that the average queue length is located approximately around the desired target value, even in the presence of different congestion levels. This is obtained by dynamically adjusting the maximum drop probability of RED $\left(p_{\max }\right)$. ARED observes the average queue length in order to infer whether to be more or less aggressive with respect to the standard RED implementation. ARED makes use of two thresholds $\left(t h_{\min }\right.$ and $\left.t h_{\max }\right)$, exactly like RED. These thresholds are set to half the target queueing delay and one and a half the target queueing delay, accordingly to the rules in [9]. If the average queue length goes below the $t h_{\min }$ threshold, then early detection is too aggressive. Similarly, if the average queue length goes above $t h_{\max }$ threshold, then early detection is too conservative. By using additive increase and multiplicative decrease policy (similarly to many TCP congestion control algorithms), ARED adaptively tune the $p_{\max }$ probability in the way that the average queue length can be targeted to the middle point between the thresholds $t h_{\min }$ and $t h_{\max }$ ARED updates $p_{\max }$ periodically after every interval (500 $\mathrm{ms}$ by default).

CoDel. Controlled Delay (CoDel) [10] is a timestamp based AQM that use the queuing latency (i.e. the delay experienced by a packet in the queue) instead of the more commonly used queue length. Codel has been proposed recently to overcome the bufferbloat problem by limiting the queueing delay to a specific target delay and dropping packets on the departure (dequeuing time).

The timestamps are used to track the minimum queuing delay that packet experience in a defined interval, set to $100 \mathrm{~ms}$ by default, When the minimum queuing delay, measured during an interval, has exceeded the target delay value, a packet is dropped from the tail and the next dropping time in computed by a control function based on the amount of previous drops. When the queuing delay goes below target delay, CoDel stops dropping packets and exits the dropping state. CoDel only enters the dropping state when the minimum queuing delay has exceeded target delay for an interval long enough to absorb normal packet bursts (100 ms by default). This ensures that a burst of packets will not experience packet drops as long as the burst can be cleared from the queue within a reasonable period. A particular characteristic of CoDel is that it drops packets at the dequeue stage (packet departure).

GREEN. Generalized Random Early Evasion Network 
(GREEN) [11] is a rate-based AQM that operates by using a drop probability that depends on the flow RTT, on the number of active flows, and on the Maximum Segment Size (MSS) encountered during a sampling time.

$$
p_{\text {drop }}=\left(\frac{N \cdot M S S \cdot C}{L \cdot R T T}\right)^{2} .
$$

Equation 1 show the probability function computed by GREEN at each packet enqueue. With ten flows, the GREEN probability function is almost flat, with the consequence that GREEN behaves in the same way for flows at 50ms of RTT and flows at 500ms of RTT. With 100 flows, which is already a flows number limit for IoT environments, the RTT range in which GREEN smooths the traffic is circa [40-200] ms which poses GREEN to be a possible candidate for this kind of networks. With a very high number of flows, i.e. 1000 flows, GREEN starts to severely drop packets making this AQM an algorithm not suitable for backbone networks. Moreover, GREEN works by registering the RTT of each flow and the flows amount during a sample time, which is again an infeasible technique on the backbone network.

PIE. Proportional Integral controller Enhanced (PIE) [12] randomly drops a packet at the onset of congestion. It shares similarities with CoDel, as for instance, it uses queuing latency instead of queue length, and in particular it uses trend of latency over time to determine the congestion level. Every $t_{\text {update }}$ time units, PIE estimates the current queueing delay by using the previous estimation of the queueing delay and the target queueing delay (a parameter of PIE). The drop probability calculation incorporates the direction in which the delay is moving by employing a classic Proportional Integral (PI) controller design. PIE, differently from CoDel, does not require timestamping due to its drop policy that works on the packet arrival (enqueueing time), instead of working on the packet departure (dequeuing time), resulting in a more lightweight mechanism.

PINK. Proactive Injection into ACK (PINK) [13] is a novel rate-based and RTT-independent AQM algorithm. It uses timestamps like CoDel but in this way: it marks packets on the direct path and wait for the ACK packets coming back on the reverse path. It then updates the advertisement window used by TCP for flow control imposing a rate which is fair on the bottleneck link and depends on the flow RTT. PINK calculates (for each ACK packet) the value for the advertised RCV.WND, which will be then written in the header. The key concept is that this value is calculated so as to prevent bottleneck congestion using the formula:

$$
R C V . W N D_{i}=B_{i}=\left\lfloor\frac{B W \cdot R T T_{i}^{\text {min }} \cdot c}{n}\right\rfloor
$$

Considering that $\frac{B W}{n}$ is the bandwidth allocation given to each active flow $i$, in Equation $2 B W$ is the bottleneck bandwidth, $n$ is the current number of active flows, $R T T_{i}^{\text {min }}$ is the minimum RTT calculated by PINK for the flow $i$ while $R C V . W N D_{i}$ is the new RCV.WND value to write in the

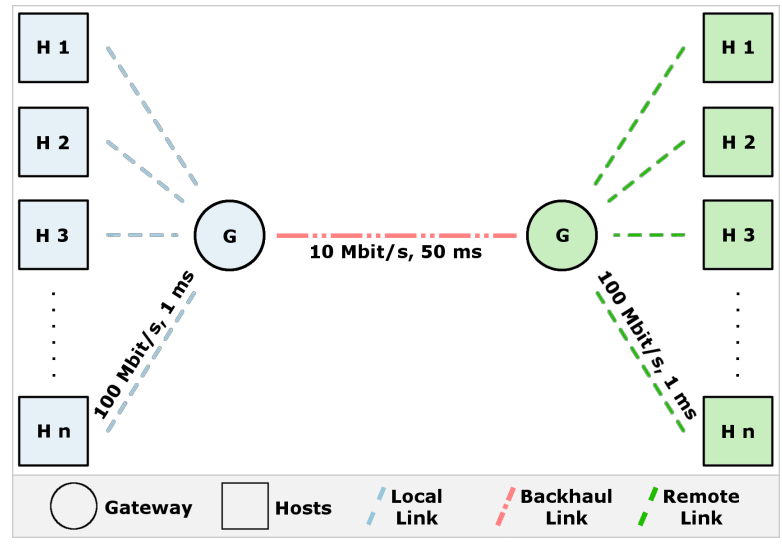

Fig. 1. Network dumb-bell topology.

TABLE I

EXPERIMENTAL NETWORK SETUP PARAMETERS

\begin{tabular}{|c|c|}
\hline Model & ns3-2.24 \\
\hline $\begin{array}{c}\text { Access/Remote } \\
\text { Network }\end{array}$ & Ethernet $100 \mathrm{Mbit} / \mathrm{s}$ \\
\hline $\begin{array}{c}\text { Bottleneck } \\
\text { Network }\end{array}$ & Ethernet $10 \mathrm{Mbit} / \mathrm{s}$ \\
\hline Base RTT & $100 \mathrm{~ms}$ \\
\hline Multi RTT & $100,150,200,250 \mathrm{~ms}$ \\
\hline MTU & 500 Bytes \\
\hline MSS & 440 Bytes \\
\hline Active Clients & $2,4,8,16,32,64$ \\
\hline AQM & $\begin{array}{c}\text { DropTail, ARED, CoDel, } \\
\text { GREEN, PIE, PINK }\end{array}$ \\
\hline
\end{tabular}

TCP window field, equal to $B_{i}$ that represents the burst value of flow $i$. The constant parameter $c$, called the "exploitation parameter", ranges from 0 to 1 and represents the channel exploitation factor. PINK incur in the same drawbacks of GREEN. It needs to trace RTT and recompute TCP ACK Checksum, this is a feasible operation only for an access network, with higher number of flows or higher data rates (backbone networks), PINK must be paired with specialized hardware [28].

\section{Simulation EnViRonment}

To highlight the effect of congestion control obtained by selecting the proper AQM in multiple environments, we developed a testbed within the ns 3 network simulator. The network topology used is the classical dumb-bell topology reported in

TABLE II

AQM TUNABLE PARAMETERS

\begin{tabular}{|c|c|}
\hline AQM & Parameters \\
\hline \hline DropTail & Queue Size $=125 \mathrm{~KB}=\mathrm{BW} * \mathrm{RTT}$ \\
\hline \multirow{2}{*}{ ARED } & interval_time $=500 \mathrm{~ms}$ \\
& $\alpha=0.01$ \\
& $\beta=0.9$ \\
\hline \multirow{2}{*}{ CoDel } & interval_time $=100 \mathrm{~ms}$ \\
& target_delay $=5 \mathrm{~ms}$ \\
\hline GREEN & interval_time $=500 \mathrm{~ms}$ \\
\hline & update_time $=30 \mathrm{~ms}$ \\
PIE & target_delay $=20 \mathrm{~ms}$ \\
& $\alpha=0.125$ \\
& $\beta=1.25$ \\
\hline PINK & exploitation constant $c=0.98$ \\
\hline
\end{tabular}




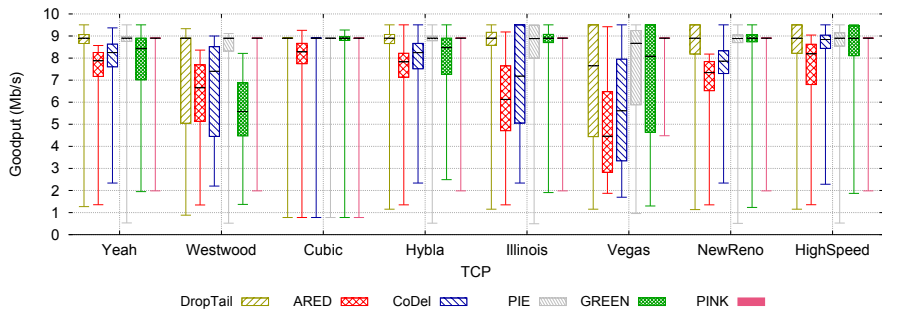

Fig. 2. Aggregate application goodput (per 1-sec intervals). TCP and AQM cross check with 16 active nodes (average congestion) with $\mathrm{RTT}_{\text {base }}=100 \mathrm{~ms}$; bottom and top of whisker-box plots show the 10th and the 90th percentiles, respectively.

Figure 1 and compliant to the topology used in many of the related works on congestion control study like [3], [24]-[26], [29]-[34].

The parameters used in our simulations are summarized in Table I; they represent two distinct access networks, connected with a bottleneck link that is accessible to the end hosts of each network through a border gateway. Testbed source code, with instructions to reproduce our results, is available at [35].

With this topology, it is possible to have a comprehensive view of the synergy between TCP and AQM algorithms, in particular, to highlight the AQM which is most resilient to the TCP variations on congestion. Because each AQM needs some parameter setting, in Table II we listed the tunable parameters of each one, together with their default values.

We ran various experiments consisting of a set of backlogged TCP flows, created between pairs of end hosts in which sender and receiver belong to different networks. Common parameters for the experiments are the bottleneck maximum queue size, set equal to the network Bandwidth Delay Product of the bottleneck link (125 KB), and their duration, set to 300 seconds. We describe comparisons and outcomes, along with specific experiment parameters, in the next Section.

\section{Performance}

In this Section, we analyze the performance of the 6 AQM algorithms DropTail, ARED, CoDel, PIE, GREEN and PINK in the presence of a specific TCP variant, like YeAH, Westwood, Cubic, Hybla, Illinois, Vegas, New Reno and HighSpeed or in the presence of multiple TCP variants on the network. We evaluate performance of goodput achieve during the experiments, the per-packet RTT distributions (latency), the gateway queue backlog, the long-run flow fairness and a simple drop analysis.

We start our analysis with a simple experiment in which each AQM is deployed on an averagely congested network composed by 16 simultaneously active TCP flows, using the same TCP variant, continuously backlogged for 300 seconds of simulation. Figure 2 shows the achieved goodput of each AQM-TCP couple on this experiment. This figure of merit, like many other in this paper, is represented with candlesticks in order to have, in a single graph, both a measure of the average value and its variance, to give an idea about the distribution of the values. It is immediately possible to note how delay-based

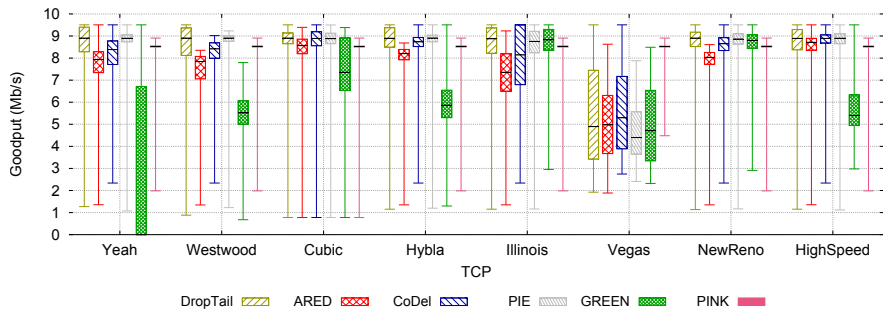

Fig. 3. Aggregate application goodput at the bottleneck link (per 1-sec intervals). TCP and AQM cross check with 64 active nodes (high congestion) with $\mathrm{RTT}_{\text {base }}=100 \mathrm{~ms}$; bottom and top of whisker-box plots show the 10th and the 90th percentiles, respectively.

TCP variants, like Vegas and Illinois, and the Westwood one, based on bandwidth estimation, suffer the channel exploitation problem resulting in a lower goodput in general. With these TCP protocols all the AQMs, excluding PINK, manifest a performance degradation if compared with other TCP with the same AQM. An interesting result is that both ARED and CoDel seem to be more TCP dependent concerning the other AQMs with almost the same trend moving from a TCP variant to another. The PIE algorithm, instead, is more stable and always as good as DropTail, which has to be considered the benchmark from a goodput point of view; only the variance of the PIE' candlestick is affected by the three most unlikely TCP variants Vegas, Illinois and Westwood. The GREEN algorithm reaches its worst-case performance if paired with TCP Westwood, while maintains high goodput values with the other TCPs. We terminate the discussion on Figure 2 by noticing the high stability of PINK, with high goodput value, thick candlestick variation and a TCP-independent property that will also be re-proposed in the other figures of merit reported in this section.

An interesting evolution from the experiment of Figure 2 is to stress the network congestion by increasing the number of active flows. This is what happen in Figure 3, where a high congested environment with 64 active TCP connections is presented. By moving from the previous Figure to this one, it is possible to notice a strong degradation of GREEN' performance. Excluding the GREEN AQM, all the queue manager algorithms achieve better results in a higher congestion environments. The exceptions are TCP Vegas, which continues to suffer goodput performance metric, and PINK, which maintain high and stable TCP-independent values but slightly lower those obtained in the less congested environment. This is reasonable considering that PINK, from its formula of bandwidth subdivision, suffers the nodes increment due to rounding errors and the difference between data payloads and frames, synthesized in a single parameter $c$.

Starting from the first experiment of Figure 2, another interesting evolution is to introduce different RTT flows. Instead of having a $100 \mathrm{~ms}$ of $\mathrm{RTT}_{\text {base }}$, in this simulation we have four class (equally distributed) of RTT with 100, 150, 200 and 250 ms. Figure 4 summarizes the goodput achieved in this harsh multi-RTT environment. The first thing to note is that ARED is the AQM with the highest performance degradation due to the 


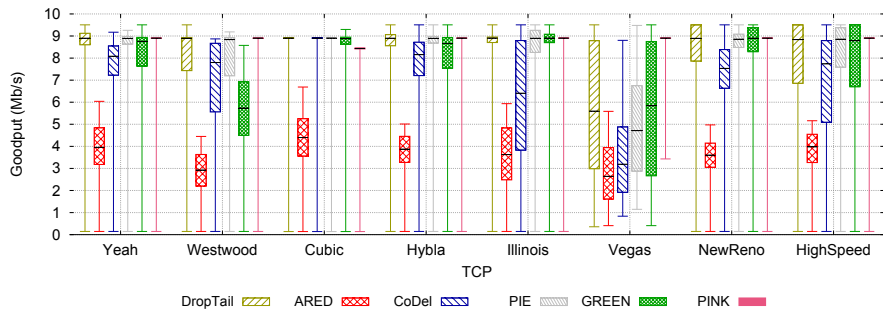

Fig. 4. Aggregate application goodput at the bottleneck link (per 1-sec intervals). TCP and AQM cross check with 16 active nodes (average congestion) with $\mathrm{RTT}_{\text {base }}$ in [100 - 250]ms interval; bottom and top of whisker-box plots show the 10th and the 90th percentiles, respectively.

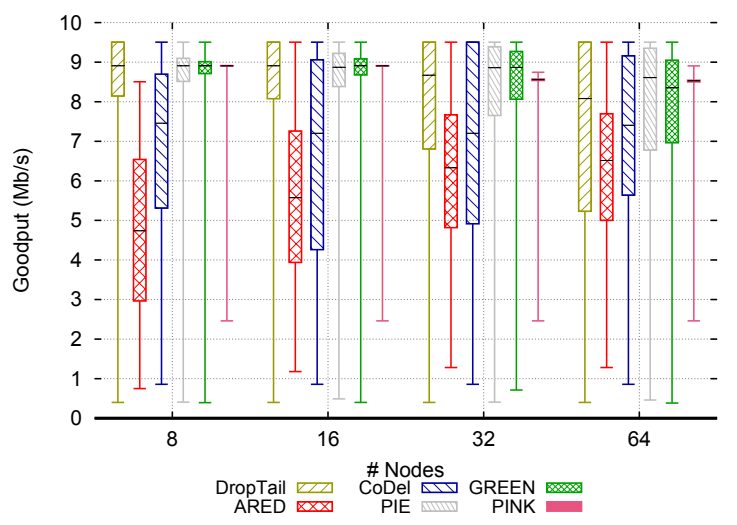

Fig. 5. Application goodput at the bottleneck link (per 1-sec intervals). MultiTCP with different congestion levels and RTT $_{\text {base }}=100 \mathrm{~ms}$; bottom and top of whisker-box plots show the 10th and the 90th percentiles, respectively.

variety of RTTs, a degradation that is almost independent of the TCP variant. Even CoDel degrades a bit with this figure of merit, where only with the Cubic TCP variant preserves a high goodput and a high RTT resiliency. Finally, PIE and PINK appear to be very robust to the RTT variation with stable goodput levels comparable to the ones reached in Figure 2.

These three simulations regarding the goodput achieved by the system have been introduced to highlight the dependency of the AQM performance to the TCP protocol, the network congestion level, and the RTT distribution.

Goodput. In this second set of experiments, the congestion level varies from 8 up to 64 active flows, following the powers of 2 . All the 8 TCP variants are used, each TCP variant with the same number of flows (i.e. 1 flow per each TCP variant in the simulation with 8 active flows and 8 flows per each TCP variant in the simulation with 64 active flows). All the TCP flows are simultaneously active and continuously backlogged for 300 seconds of simulation.

Figure 5 shows the result, regarding achieved goodput, of this second set of simulation. It is easy to notice immediately how CoDel and particularly ARED suffer the presence of multiple TCP variants together. While CoDel is stable as a function of the network congestion, ARED suffers from averagely congested networks and reaches performance comparable to CoDel when the congestion increase. Both DropTail and GREEN manifest a good and stable performance with a slightly degrade with 64 active nodes. PIE and PINK maintain

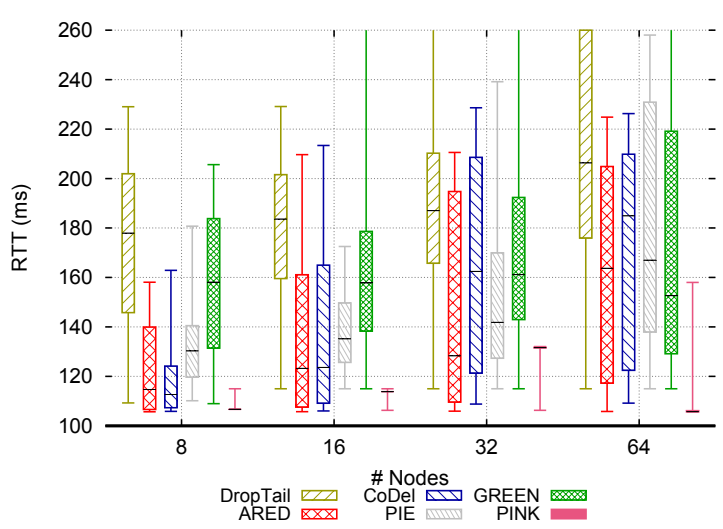

Fig. 6. Flows' RTT variation (per 1-sec intervals). Multi-TCP with different congestion levels and $\mathrm{RTT}_{\text {base }}=100 \mathrm{~ms}$; bottom and top of whisker-box plots show the 10th and the 90th percentiles, respectively.

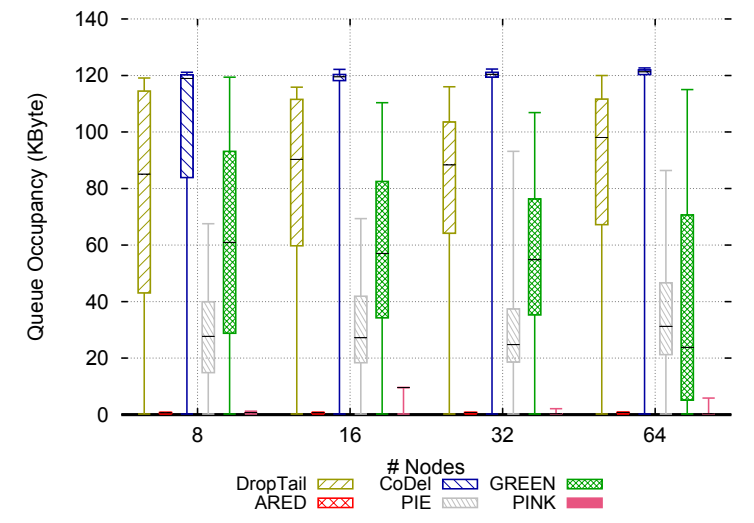

Fig. 7. Bottleneck queue backlog level (per 1-sec intervals). Multi-TCP with different congestion levels and $\mathrm{RTT}_{\text {base }}=100 \mathrm{~ms}$; bottom and top of whiskerbox plots show the 10th and the 90th percentiles, respectively.

remarkable results even in this challenging scenario. It is clearly undesirable, in several applications, to have a higher goodput if it comes at the cost of an increased application delay: in the following, we investigate the delay perceived at the application level (through an RTT analysis).

Queue backlog and RTT analysis. During the same simulation used to provide Figure 5, we have also collected information about RTT variation and gateway' queue backlog level reported in Figure 6 and Figure 7 respectively.

The simple DropTail, as can be expected, manifest the worst performance regarding latency, with RTT variations that double the $\mathrm{RTT}_{\text {base }}$ of the network. At the same time, it forces the queue to be almost full for all the experiment duration. ARED, which was suffering a bit regarding the goodput performance, here manifest a very good behavior in terms of queue occupancy and also a good latency stability that anyway degrades as a function of the network congestion as can be noticed in Figure 6. The same trend of latency and RTT variation is reported by CoDel, which has average values slightly higher than ARED. A characteristic of CoDel is the counter-intuitive high level of queue backlog, this is simply due to its drop policy that is triggered during dequeue stage; 
this behavior is traduced in a queue backlog level that is only "virtually" high because the drops have still to be performed. GREEN has an interesting opposite trend; it reduces both the average latency and the queue backlog level as a function of the number of active flows while increasing the variance of the values, noticeable with wider candlestick box. PIE suffers at the beginning with low network congestion levels ( 8 and 16 flows) the comparison with ARED and CoDel in terms of latency, but it recovers increasing the network congestion (32 and 64 nodes) thanks to a trend more scalable as a function of the congestion level. Its queue backlog level in Figure 7 in fact is very stable, both for the average value and for the values distribution, remarking the PIE scalability. The performance of PINK is very promising with both the figure of merit almost at the optimal values.

All the experiments are available in [35] with more figures of merit not included here for space limitation.

\section{CONCLUSIONS}

In this paper, we proposed an extensive analysis of the performance of different AQM algorithms in IoT networks. Many figures of merit have been computed to enrich the analysis, as the flows' goodput, the RTT variation ad well as the queue backlog level at the gateway. We initially showed how the TCP variants affect the global performance, together with possible multi-RTT flows and the network congestion level. We then moved to multi-TCP and more realistic IoT heterogeneous environment.

Results have shown how a "one size fit all" solution does not exist, as usually happen in the networking field. ARED gave it best performance in a high congested environment but suffer in an unacceptable way in the presence of multi-RTT flows. CoDel has revealed to be more resilient to this issue and, like ARED, adjust the trade-off between goodput and latency performance as a function of the network congestion. PIE has been surprisingly resilient to all the stress factors, it has one of the best trade-offs between channel exploitation and latency. The novel PINK AQM highlights its optimal fairness property and stable results over all the figures of merit.

\section{REFERENCES}

[1] A. Afanasyev et al., "Host-to-host congestion control for tcp," IEEE Communications Surveys Tutorials, vol. 12, no. 3, pp. 304-342, 2010.

[2] Y. Chen, X. Wang, and L. Cai, "On achieving fair and throughputoptimal scheduling for tcp flows in wireless networks," IEEE Transactions on Wireless Communications, vol. PP, no. 99, pp. 1-1, 2016.

[3] S. Stancescu, A. A. Luca, and A. Caraivan, "Tcp-aqm algorithms which combat the computer networks' congestion," in Telecommunications Forum Telfor (TELFOR), 2014 22nd, Nov 2014, pp. 182-185.

[4] N. Cardwell et al., "Bbr: Congestion-based congestion control," Communications of the ACM, vol. 60, no. 2, pp. 58-66, 2017.

[5] P. Schulz et al., "Latency critical iot applications in 5g: Perspective on the design of radio interface and network architecture," IEEE Cотmиnications Magazine, vol. 55, no. 2, pp. 70-78, 2017.

[6] Y. Lu et al., "Sdtcp: Towards datacenter tcp congestion control with sdn for iot applications," Sensors (Switzerland), vol. 17, no. 1, 2017.

[7] W. Li et al., "Learning-based and data-driven tcp design for memoryconstrained iot," 2016, pp. 199-205.

[8] R. Duan, X. Chen, and T. Xing, "A qos architecture for iot," 2011, pp. $717-720$.

[9] S. Floyd et al., "Adaptive red: An algorithm for increasing the robustness of red's active queue management," Preprint, available at http://www. icir. org/floyd/papers. html, 2001.
[10] K. Nichols and V. Jacobson, "Controlling queue delay," Communications of the ACM, vol. 55, no. 7, pp. 42-50, 2012.

[11] W. chun Feng, A. Kapadia, and S. Thulasidasan, "Green: proactive queue management over a best-effort network," in GLOBECOM 'O2. IEEE, Nov 2002, pp. 1774-1778.

[12] R. Pan et al., "Pie: A lightweight control scheme to address the bufferbloat problem," in High Performance Switching and Routing, 2013 IEEE International Conference on. IEEE, 2013, pp. 148-155.

[13] C. Grazia et al., "Pink: Proactive injection into ack, a queue manager to impose fair resource allocation among tcp flows," in 2015 IEEE WiMob, Oct 2015, pp. 132-137.

[14] S. Ha, I. Rhee, and L. Xu, "Cubic: a new tcp-friendly high-speed tcp variant," ACM SIGOPS, vol. 42, no. 5, pp. 64-74, 2008.

[15] S. Floyd, "Limited slow-start for tcp with large congestion windows," 2004.

[16] C. Caini and R. Firrincieli, "Tcp hybla: a tcp enhancement for heterogeneous networks," International Journal of Satellite Communications and Networking, vol. 22, no. 5, pp. 547-566, 2004.

[17] S. Liu, T. Başar, and R. Srikant, "Tcp-illinois: A loss- and delay-based congestion control algorithm for high-speed networks," Performance Evaluation, vol. 65, no. 6-7, pp. 417 - 440, 2008.

[18] S. Floyd, A. Gurtov, and T. Henderson, "The newreno modification to tcp's fast recovery algorithm," 2004.

[19] G. Hasegawa, K. Kurata, and M. Murata, "Analysis and improvement of fairness between tcp reno and vegas for deployment of tcp vegas to the internet," in Network Protocols Conference, 2000, pp. 177-186.

[20] L. A. Grieco and S. Mascolo, "Performance evaluation and comparison of westwood+, new reno, and vegas tcp congestion control," $A C M$ SIGCOMM Com. Com. Review, vol. 34, no. 2, pp. 25-38, 2004.

[21] A. Baiocchi, A. P. Castellani, and F. Vacirca, "Yeah-tcp: yet another highspeed tcp," in in Proc. PFLDnet, ISI, February 2007, pp. 37-42.

[22] R. Adams, "Active queue management: A survey," IEEE Communications Surveys Tutorials, vol. 15, no. 3, pp. 1425-1476, Third 2013.

[23] H. Rahman, K. Giridhar, and G. Raina, "Performance analysis of compound tcp with aqm," 2013, pp. 492-499.

[24] G. Raina et al., "Stability and performance analysis of compound tcp with rem and drop-tail queue management," IEEE/ACM Transactions on Networking, vol. 24, no. 4, pp. 1961-1974, Aug 2016.

[25] N. Khademi, D. Ros, and M. Welzl, "The new aqm kids on the block: An experimental evaluation of codel and pie," in Computer Communications Workshops (INFOCOM), 2014 IEEE Conference on, 2014, pp. 85-90.

[26] A. Chydzinski and A. Brachman, "Performance of aqm routers in the presence of new tcp variants," in Advances in Future Internet (AFIN), 2010 Second International Conference on, July 2010, pp. 88-93.

[27] N. Halim, N. Yaakob, and A. Isa, "Congestion control mechanism for internet-of-things (iot) paradigm,” 2016, pp. 337-341.

[28] F. Yamaguchi and H. Nishi, "Hardware-based hash functions for network applications," in Networks (ICON), 2013 19th IEEE International Conference on, Dec 2013, pp. 1-6.

[29] C. A. Grazia et al., "Integration between terrestrial and satellite networks: the ppdr-tc vision," in 2014 IEEE 10th International Conference on Wireless and Mobile Computing, Networking and Communications (WiMob), Oct 2014, pp. 77-84.

[30] - "Performance evaluation and economic modelling of ppdr communication systems," in Wireless and Mobile Computing, Networking and Communications (WiMob), 2015 IEEE 11th International Conference on, Oct 2015, pp. 75-82.

[31] M. Casoni et al., "Towards emergency networks security with per-flow queue rate management," in Pervasive Computing and Communications Workshops (PERCOM Workshops), 2014 IEEE International Conference on, March 2015, pp. 497-502.

[32] M. Casoni, C. Grazia, and M. Klapez, "An sdn and cps based opportunistic upload splitting for mobile users," in Proceedings of the EAI International Conference on CYber physiCaL systems, iOt and sensors Networks, ser. IOT360 Summit '15, 2015.

[33] _ "Enabling resource pooling in wireless networks through softwaredefined orchestration," in to appear in Communications Workshops (ICC), 2016 IEEE International Conference on, May 2016.

[34] M. Casoni et al., "Implementation and validation of tcp options and congestion control algorithms for ns-3," in Proceedings of the 2015 Workshop on Ns-3. New York, USA: ACM, 2015, pp. 112-119.

[35] "AQMvsTCP ns-3 source code," http://netlab.ing.unimo.it/sw/src.zip, September 2016. 